https://helda.helsinki.fi

\title{
Lyophilic matrix method for dissolution and release studies of nanoscale particles
}

\section{Pessi, Jenni}

2017-10-25

Pessi , J , Svanbäck , S , Lassila , I , Haeggstrom , E \& Yliruusi , J 2017 , ' Lyophilic matrix method for dissolution and release studies of nanoscale particles ' , Journal of Pharmaceutical and Biomedical Analysis, vol. 145 , pp. 549-554 . https://doi.org/10.1016/j.jpba.2017.07.017

http://hdl.handle.net/10138/307963

https://doi.org/10.1016/j.jpba.2017.07.017

cc_by_nc_nd

acceptedVersion

Downloaded from Helda, University of Helsinki institutional repository.

This is an electronic reprint of the original article.

This reprint may differ from the original in pagination and typographic detail.

Please cite the original version. 
1 Lyophilic matrix method for dissolution and release studies of nanoscale 2 particles

3 Jenni Pessi ${ }^{1 *^{*},}$, Sami Svanbäck ${ }^{1 \ddagger}$, Ilkka Lassila ${ }^{2}$, Edward Hæggström ${ }^{2}$, Jouko Yliruusi ${ }^{1}$

$4{ }^{1}$ Division of Pharmaceutical Chemistry and Technology, Faculty of Pharmacy, University of 5 Helsinki, Viikinkaari 5e, 00790 Helsinki, Finland

$6 \quad{ }^{2}$ Division of Material Physics, Department of Physics, University of Helsinki, Gustaf Hällströmin 7 katu 2a, 00560 Helsinki, Finland

$8 *$ Corresponding author. Tel.: +358-50-381-0225.

$9 \quad$ E-mail address: jenni.pessi@helsinki.fi

10 \$ These two authors contributed equally.

\section{Highlights:}

- The lyophilic matrix (LM) method for dissolution and release studies of powders, nanoscale particles, and particulate systems is introduced.

- LM method avoids major issues encountered with current dissolution methods such as the membrane effect and dispersion of the non-dissolved particles.

- LM method permits rapid contact with the dissolution medium, enables separating the dissolved species from the non-dissolved particles, and clearly displays the different dissolution rate of different size particles.

\section{Abstract}

We introduce a system with a lyophilic matrix to aid dissolution studies of powders and particulate systems. This lyophilic matrix method (LM method) is based on the ability to discriminate between non-dissolved particles and the dissolved species. In LM method the test substance is embedded in a thin lyophilic core-shell matrix. This permits rapid contact with the dissolution medium while minimizing dispersion of non-dissolved particles without presenting a substantial membrane effect. The method produces realistic dissolution and release results for particulate systems, especially those featuring nanoscale particles. By minimizing method-induced effects on the dissolution profile of nanopowders, the LM method can overcomes shortcomings associated with current dissolution test methods. 


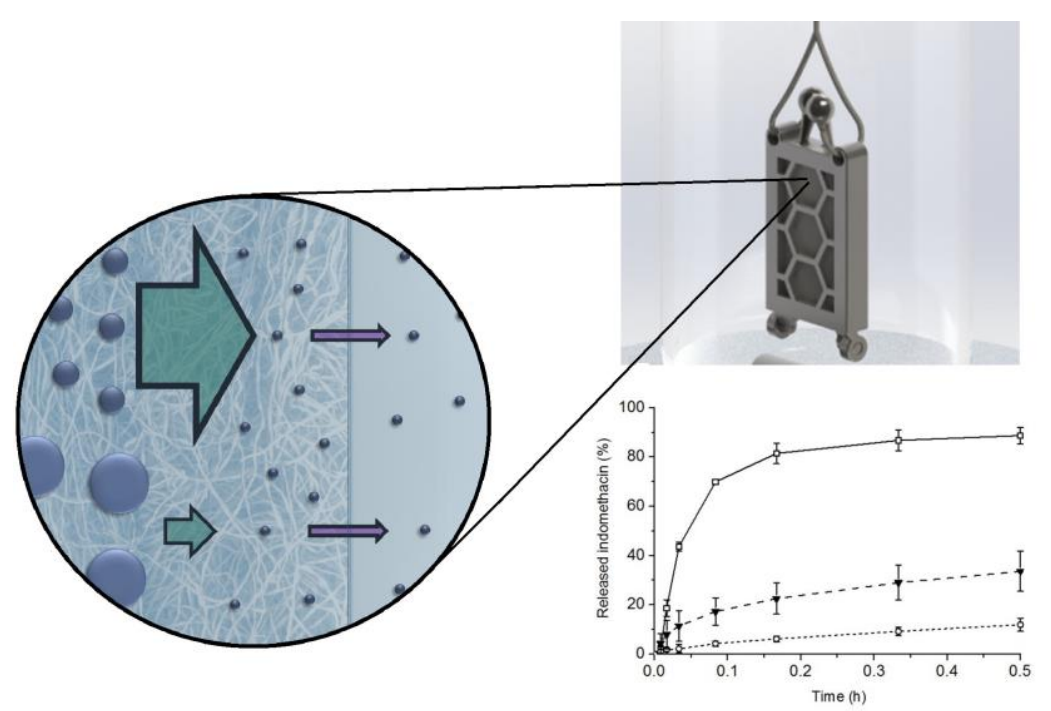

Keywords:

Dissolution; Dissolution rate; Drug release; Particulate systems; Nanoparticles; Lyophilic matrix method

\section{Introduction}

The dissolution rate of a drug is a physico-chemical property to be determined and modified during drug discovery and development $[1,2]$. For example, reducing the particle size to the nanoscale increases the dissolution rate and thus the bioavailability of poorly water-soluble drugs in classes II and IV of the Biopharmaceutics Classification System [3-5]. The dissolution rate of nanoscale particles correlate with the performance and quality of a formulation featuring nanoparticles [3]. Hence to assess the impact of nanonizing a poorly water-soluble drug, one needs reliable dissolution rate data of nanoparticulate systems. Such data could allow one to predict realistic in vitro - in vivo (IVIV)-correlation and facilitate determination of dose in animal experiments [6-8].

Current methods for investigating dissolution rates of nanoscale particles include the United States Pharmacopoeia (USP) I (basket), II (paddle), and IV (flow-through) methods, as well as modifications thereof, membrane diffusion methods (such as the dialysis methods), and sample and separate methods (such as centrifugal ultrafiltration) [6, 7, 9-14]. Additionally, dissolution rates of nanoparticles have been determined from tablets and admixtures using gel matrices $[15,16]$.Often, the measured values reflect features of the dissolution test device, equipment or method, rather than the nanoparticle properties. 
The main issues with the current methods include: dispersion of non-dissolved particles, hydrodynamics-induced variability, membrane effects caused by diffusion barriers (e.g. gelatin, filters, or dialysis membranes), clogging and breaking of filters, sensitivity to flow and location in the dissolution vessel, as well as migration of nanoparticles to interfaces (e.g. wetting issues, floating, or adhesion) [17-24]. The UPS methods were not designed for dissolution studies of nanoscale particles and thus produce unrealistic results [13, 17]. Dispersion and the consequent overestimation of nanoparticle dissolution rates in the USP I and II methods occur when the location of the particles is not fixed. In the USP IV method dispersion occurs when a too large filter pore size is used [6]. On the other hand, constraining diffusion of the dissolved species by membranes or encapsulation, leads to measurement of the quality of the diffusion barrier rather than the nanoparticle dissolution, and often to underestimating the dissolution rate $[17,19,25,26]$. Using tablets or admixtures may alter the physical form of the drug during the tableting or mixing processes, and they may detach particles from the tablet surface during the dissolution process, or induces a diffusional barrier [15, 16]. Accordingly, there is a need for new methods and devices for determining dissolution rates of nanoparticles.

\section{Materials and methods}

\subsection{Chemicals}

Indomethacin (Hawkins, USA) was used as poorly water-soluble model compound in the dissolution experiments and poloxamer 188 (BASF Co., Germany) was used as stabilizer. The chemicals used for preparing the media for the dissolution studies were monopotassium phosphate (Riedel-de Haën, Germany), sodium phosphate dibasic (Sigma-Aldrich, USA), and 5M sodium hydroxide (VWR Chemicals BDH Prolabo, EC). All chemicals employed in the experiments were of analytical grade and used as received.

\subsection{Structure of the device}

The device used in the dissolution experiments comprised a lyophilic matrix, a cage, a vessel, and a mixing/heating plate (Fig. 1). The matrix has a core-shell structure comprising a core matrix, that contained the particles of the test substance, and a surrounding shell matrix. The matrix material of both core and shell matrices is cotton (100\% cotton, Curatex GmbH, Germany). The shell matrix consists of four layers of water jet-pressurized cotton with a dry specific surface weight of $5 \pm 0.2$ $\mathrm{mg} / \mathrm{cm}^{2}$. Cotton was selected as matrix material due to its unique properties; hollow cellulose fibers, high wet strength, inert nature, and substantial ability to absorb water-based media. The custom 
designed stainless steel cages (depth $3 \mathrm{~mm}$ x height $26 \mathrm{~mm}$ x width $16 \mathrm{~mm}$ ) were 3D printed with selective laser sintering (Mlab Cusing, Concept Labs, Germany). The cage maintained the desired matrix geometry and provided a fixed diffusion distance.
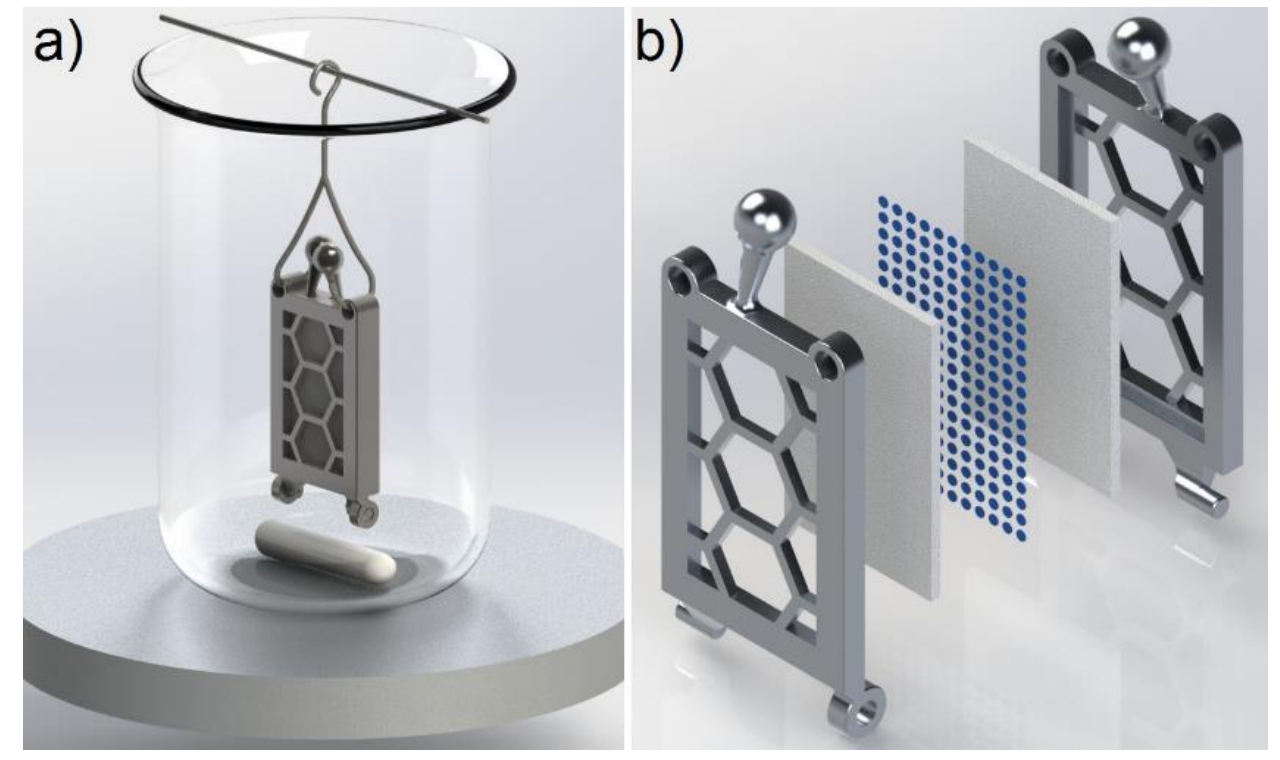

Fig. 1. a) LM method test setup and b) core-shell structure within the LM device, blue dots represent the core matrix containing the particles surrounded on all sides by the shell matrix and cage.

\subsection{Characterization of the matrix}

\subsubsection{Matrix-medium interaction}

The cotton matrix was examined prior to, during, and after medium exposure with light microscopy (Leica DMLB, Leica Microsystems Wetzlar, Germany) with a magnification of $200 \mathrm{x}$, and prior to, and after medium exposure with scanning electron microscopy (SEM, Quanta ${ }^{\mathrm{TM}} 250$ FEG, FEI Inc., USA) with a magnification of $500 \mathrm{x}$, voltage of $5.00 \mathrm{kV}$, spot size of 3.0, sputter coated with a 5-nmthick platinum layer (Q150T Quomm, Beijing, China). The water up-take properties of the matrix were investigated with a fast camera $(1200 \mathrm{fps}$, Casio Exilim High-speed EX-FI1, Casio, Japan) and by weighing the matrix prior to and after exposure to the medium.

\subsubsection{Drug-matrixn interaction}

The partitioning of the model compound between the matrix and medium was examined by partition coefficient and inverse partitioning coefficient studies. First, the retention of the model compound within the matrix was examined. This was done by partition coefficient tests, where the matrix containing $1 \mathrm{mg}$ of bulk indomethacin was immersed in the medium, and collected after 22 hours. 
101 The indomethacin retained in the matrix was determined by immersing the matrix into fresh medium for 22 hours. This procedure was conducted with three parallel experiments in $\mathrm{pH} 5.5$ and $\mathrm{pH} 7.4$ phosphate buffer media [27] at $37.0 \pm 0.5^{\circ} \mathrm{C}$ with a stirring rate of $180 \mathrm{rpm}$ (IKA RT $15 \mathrm{P}$, IKA Werke GmbH \& CO. KG, Germany). The concentration of the medium was determined after first and second immersion at time point of $22 \mathrm{~h}$. The concentration of the samples was analyzed with high performance liquid chromatography (HPLC Thermo System Products, Agilent 1200 Infinity Series, Agilent Technologies, Germany), using a Discovery C18 column $(4.6 \times 150 \mathrm{~mm}, 5 \mu \mathrm{m}$, Supelco, USA), $1.5 \mathrm{~mL} / \mathrm{min}$ flow rate with a mobile phase consisting of 60:40 (V/V) acetonitrile (ACN) and $0.2 \%$ ortophosphoric acid $\left(\mathrm{H}_{3} \mathrm{PO}_{4}\right)$ in water (MilliQ), operating at $30{ }^{\circ} \mathrm{C}$ with detection at $270 \mathrm{~nm}$. The standard curve for indomethacin quantification was acquired from triplicate samples of indomethacin concentrations between $0.08 \mathrm{mg} / \mathrm{L}$ and $500 \mathrm{mg} / \mathrm{L}\left(\mathrm{R}^{2}=0.999\right)$.

Second, the partitioning of the dissolved species into the matrix was examined. This was done by inverse partition coefficient tests, where an empty matrix was inserted into medium with saturated concentration of the model compound. Test was conducted in triplicate in phosphate buffer media with $\mathrm{pH}$ of 5.5 and 7.4. The empty matrices were inserted into the medium every 5 minutes and the test run was 20 minutes. The concentration of the medium was monitored online using in-situ fiberoptic UV monitoring (Opt-Diss 410, Distek, Inc., USA) using probes with a path-length of $5 \mathrm{~mm}$, exposure time of $44 \mathrm{~ms}$ (4 scans/data point) at an analytical wavelength of $320 \mathrm{~nm}$.

\subsection{Drug release studies}

\subsubsection{Preparation and characterization of the particles}

A nanosized fraction, two sieved particle size fractions, and bulk indomethachin were tested with the LM method. Nanosuspension was prepared by milling with a Fritsch Pulverisette 7 Premium ball mill (Fritsch GmbH, Germany) to obtain particles for the experiments. Nanoparticles for the LM method were prepared of $2 \mathrm{~g}$ indomethacin suspended in solution containing $5.0 \mathrm{~mL} 0.24 \mathrm{~g} / \mathrm{mL}$ poloxamer 188 solution ( $60 \mathrm{wt} \%$ relative to the drug amount) and $5.0 \mathrm{~mL}$ water (milliQ), and by grinding at 850 rpm in 5 cycles of 3 min using $60 \mathrm{~g}$ milling pearls (zirconium oxide, diameter $1 \mathrm{~mm}$ ). The particle size distribution in the nanosuspension was determined with a Zetasizer Nano SZ (Malvern Instruments Ltd., UK).

The bulk indomethacin was divided into two fractions using a sieve with an eye size of $125 \mu \mathrm{m}$ (Fritsch GmbH, Germany). The particle size of the bulk powder and the two fractions were determined from SEM images (see section 2.3.1.) $(n=300$, ImageJ freeware, National Institutes of 
133 Health, USA). The bulk powder and the two fractions were each mixed with poloxamer 188 (60 wt $\%$ 134 relative to the drug amount) to achieve physical mixtures with components identical to the 135 nanosuspension.

136

137 138 139 140 141 142 143 144 145 146 147 148

\subsubsection{Dissolution experiments}

The test substances (corresponding to $400 \mu \mathrm{m}$ of indomethacin) were distributed within the core matrix, the nanosuspension was distributed wet and left to dry. The core matrix was then placed in the matrix holder. Dissolution tests were conducted in triplicate for nanoparticles, bulk powder, and the two particle size fractions in $\mathrm{pH} 5.5$ phosphate buffer medium [27] and for nanoparticles and bulk powder in $\mathrm{pH} 7.4$ phosphate buffer medium [27]. All tests were performed under sink conditions in $100 \mathrm{~mL}$ of dissolution medium at $37.0 \pm 0.5^{\circ} \mathrm{C}$ using a stirring rate of $180 \mathrm{rpm}$ (IKA RT $15 \mathrm{P}$, IKA Werke GmbH \& CO. KG, Germany). The stirring rate and the geometry of the matrix were optimized with preliminary experiments. Aliquots of $1 \mathrm{~mL}$, subsequently replaced with the same volume of fresh medium, were taken at 12 time points: $30 \mathrm{~s}, 1 \mathrm{~min}, 2 \mathrm{~min}, 5 \mathrm{~min}, 10 \mathrm{~min}, 30 \mathrm{~min}, 1 \mathrm{~h}, 2 \mathrm{~h}, 3 \mathrm{~h}$, $4 \mathrm{~h}, 6 \mathrm{~h}$, and $22 \mathrm{~h}$. The samples were analyzed with HPLC as described in section 2.3.2. Cumulative release of indomethacin and standard deviation in three parallel samples were determined for each experiment.

\section{Results and discussion}

\subsection{Properties of the matrix}

\subsubsection{Matrix-medium interaction}

No visual changes were detected in the size, topology, and morphology of the cotton fibers as the matrix was exposed to dissolution medium, nor did the structure of the cotton change after drying (Fig. 2). When immersed into medium, the cotton matrix was wetted in $0.31 \mathrm{~s} \pm 0.10 \mathrm{~s}$. The matrix withdrew medium approximately 23 times its weight. The result of these two experiments indicate that the particles within the matrix are exposed to the medium immediately after immersion. The volume of the matrix increases when exposed to medium. However, microscope studies indicated that the single fibers do not swell when immersed into the medium. 


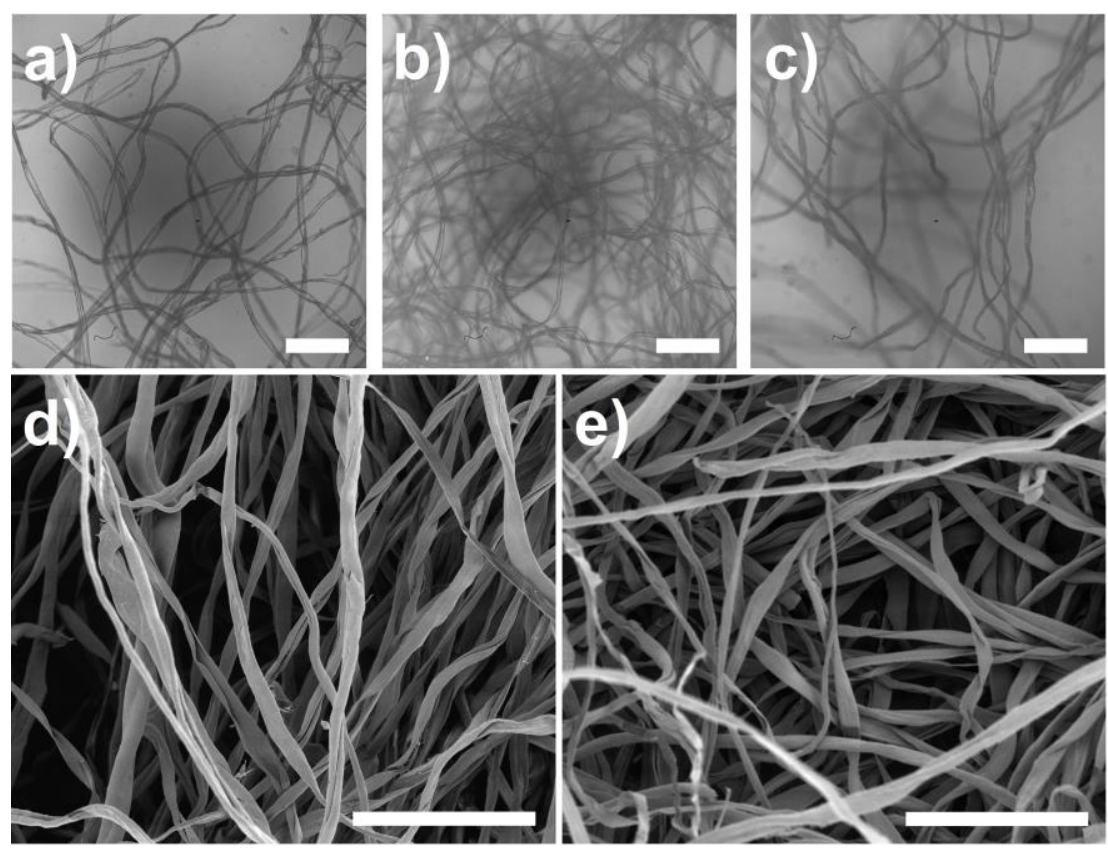

160 Fig. 2. Light microscope images of the cotton shell matrix a) prior to dissolution medium exposure, 161 b) exposed to medium, and c) after medium exposure. SEM images of the cotton d) prior to medium 162 exposure, and e) after medium exposure. Scale bars correspond to $200 \mu \mathrm{m}$.

\subsubsection{Drug-matrix interaction}

164 No retained indomethacin was found in the matrix after first 22 hours of in the partition coefficient 165 tests. The concentrations obtained were below the detection limit $(0.08 \mathrm{mg} / \mathrm{L})$ of the HPLC method. 166 This indicates that $>99.2 \%$ of indomethacin is released from the matrix and the dissolved species is 167 not significantly retained within the matrix. The inverse partition coefficient test showed no 168 detectable (detection limit: ) change in concentration when the matrix was immersed into the 169 medium with dissolved indomethacin. This indicates that the matrix does not absorb dissolved 170 indomethacin from the dissolution medium.

171 As shown in the characterization tests, the matrix is practically inert and has little effect on the total 172 quantity of indomethacin released (Table 1). As the $\mathrm{pH}$ has no effect on the partition coefficient, we 173 conclude that at least with indomethacin - a weak acid $\left(\mathrm{pK}_{\mathrm{a}} 4.5\right)$ - the change in the $\mathrm{pH}$ of the medium 174 causes no adsorption onto the fibers. The partitioning coefficient studies were conducted only in 175 regard to the dissolved species. The possible adhesion is not considered to be an issue, since the non176 dissolved particles are intended to remain within the matrix. 
Table 1

178 Summary of the investigated properties of the matrix.

\begin{tabular}{|c|c|c|}
\hline Property & Experiment & Result \\
\hline Intake of medium & Weighing & $23 \mathrm{x}$ weight of the matrix \\
\hline Wetting time & Fast camera tests & $0.31 \pm 0.10 \mathrm{~s}$ \\
\hline Impact of medium on morphology & Imaging & no impact \\
\hline $\begin{array}{l}\text { Adsorption of dissolved species to } \\
\text { matrix (from particles) }\end{array}$ & Partition coefficient & $>99.2 \%$ \\
\hline $\begin{array}{l}\text { Adsorption of dissolved species to } \\
\text { matrix (from media) }\end{array}$ & $\begin{array}{l}\text { Inverse partition } \\
\text { coefficient }\end{array}$ & $<\ldots \%$ \\
\hline
\end{tabular}

179

180

181

182

183

184

185

186

187

188

189

190

191

192

193

\subsection{Drug release studies}

\subsubsection{Particle size}

The average size of the nanoparticles was $424 \mathrm{~nm} \pm 236 \mathrm{~nm}$, and of the bulk powder $20.3 \mu \mathrm{m} \pm 30.0$ $\mu \mathrm{m}$, featuring size range of $1 \mu \mathrm{m}-272 \mu \mathrm{m}$. The average size of the small fraction was $17.4 \mu \mathrm{m} \pm$ $11.6 \mu \mathrm{m}$, and of the large fraction $22.1 \mu \mathrm{m} \pm 21.8 \mu \mathrm{m}$.

\subsubsection{Dissolution rate}

Differences in dissolution rate as function of particle size and $\mathrm{pH}$ were evident in the dissolution profiles obtained with LM method. Fig. 3 shows the cumulative release of indomethacin nanoparticles, small and large size fraction in $\mathrm{pH} 5.5$, and nanoparticles and bulk indomethacin in $\mathrm{pH} 7.4$ up to 30 min. The short lag times indicated rapid wetting of the samples and absence of any significant membrane effect. Monotonously increasing dissolution profiles and constant standard deviations indicate that the variation between aliquots is moderate, i.e. that no substantial withdrawal of particles occurred during sampling. The method was accurate with small sample quantities and differences between the dissolution rates were detected within 5 minutes from the start of the experiment as seen in the dissolution profiles. 

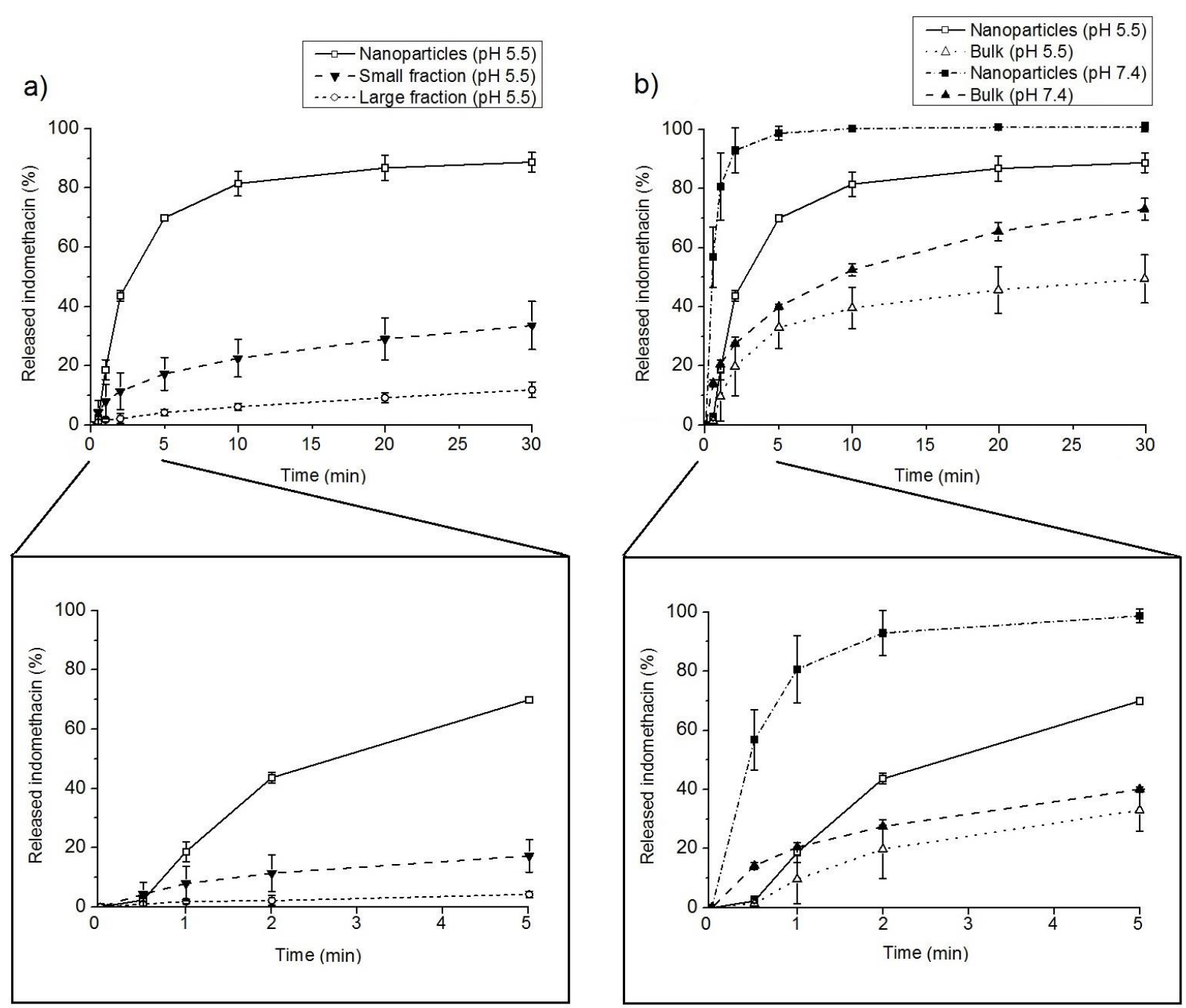

Fig. 3. Cumulative release (\%) of indomethacin a) nanoparticles, small fractions and large fraction in $\mathrm{pH} 5.5$ up to $30 \mathrm{~min}$ and $5 \mathrm{~min}$ at $37.0 \pm 0.5^{\circ} \mathrm{C}$, b) nanoparticles and bulk in $\mathrm{pH} 5.5$ and 7.4 up to 30 min and 5 min at $37.0 \pm 0.5^{\circ} \mathrm{C}$. Error bars are standard deviations of three parallel measurements.

\subsection{Principle of the LM method}

199 The key factor of the LM method is its ability to separate non-dissolved particles from dissolved 200 species and its ability to prevent dispersion of the particulates without presenting a significant 201 membrane effect. The dissolved species exit the matrix, whereas the non-dissolved particles remain within the matrix (Fig. 4). 


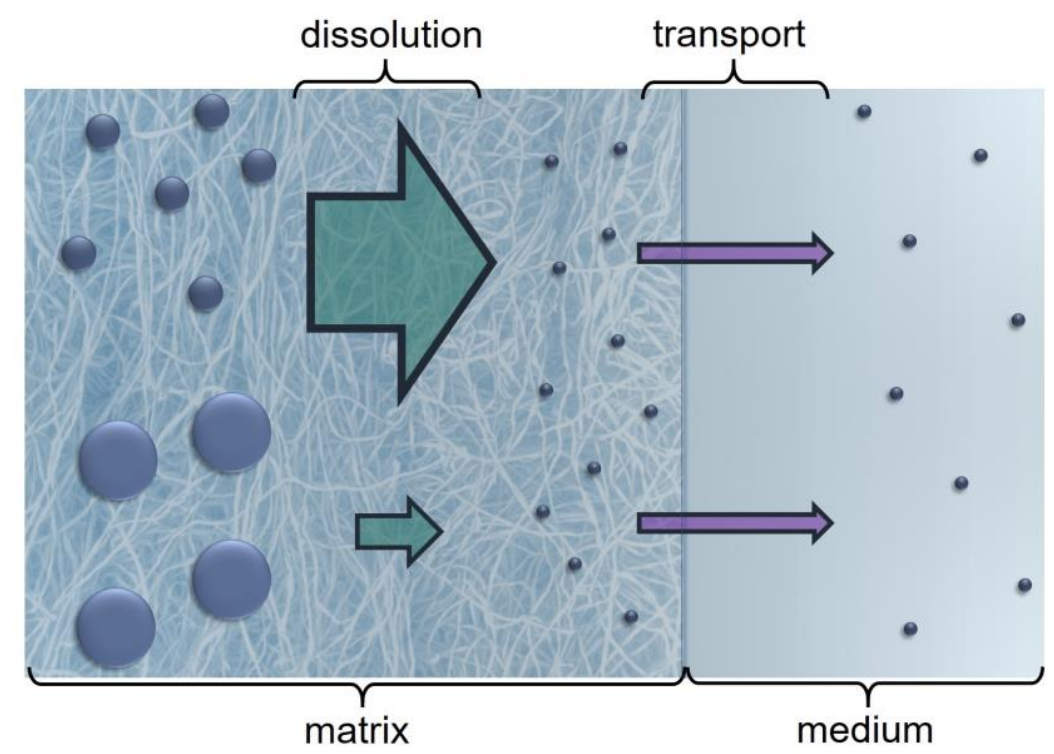

Fig. 4. Dissolved species (small spheres) diffuse promptly into the medium, whereas the diffusion velocity of the non-dissolved species (medium size and large spheres) is lower. Smaller particles (medium size spheres) dissolve to form the dissolved species faster than larger particles (large spheres).

Instead of dispersing the particles into the dissolution medium or exchanging the medium through a barrier, the matrix fixes the position of the non-dissolved particles and brings the medium to the particles. In the LM method the particles are dissolved from a stationary point in a semi 2-dimensional system under sink conditions. The efficient intake of medium, the concentration gradient, and the mild convection induced in the vessel drive the dissolved species out of the matrix. The matrix does not a form a separate compartment in the dissolution vessel and the lack of interaction between the cotton fibers and the model compound ensures that the dissolved species is not trapped in the matrix.

The dissolution of the particles within the matrix is initiated as the matrix absorbs medium. The whole particle population is wetted nearly simultaneously. The dissolution rate depends on the active surface area of the particles as described by the Nernst-Brunner equation and the radius and particle curvature as described by the Gibbs-Kelvin equation [28-30]. The equations predict that small particles dissolve faster than large ones.

Results produced by the LM method do neither overestimate the dissolution rates nor do they induce substantial lag times. The ability to produce realistic dissolution data supports early formulation development, which is valuable for evaluation of advantages gained by particle size reduction and nanonization. Small sample-to-sample variation enables producing reliable results with small interor intra-laboratory variation. Thus, it can be inferred that dissolution testing with lyophilic matrices produces realistic estimates of dissolution rates of nanoscale particles. Further studies are needed to 
prove the universal applicability of the LM method and to assess the dissolution rate for different substances as well as to verify the IVIV-correlation.

\section{Conclusions}

The LM method developed in this study is suitable for determining dissolution rates of particulate systems, especially of nanoscale particles. The method features short lag time, small sample-tosample variation, and monotonously increasing dissolution profiles. It was capable to discriminate the dissolution rates of the tested particle size fractions. The inert cotton matrix used enables release studies without any substantial membrane effect, avoiding dispersion of the non-dissolved particles, and providing rapid wetting of the sample.

\section{Acknowledgements}

J. Pessi and S. Svanbäck acknowledge the Drug Research Doctoral Program funding of the Faculty of Pharmacy, University of Helsinki. The authors thank the Electron Microscopy Unit of Institute of Biotechnology, University of Helsinki, for providing laboratory facilities, Mr. Heikki Räikkönen for technical support, and Ms. Anu Piipponen, Ms. Sanna Sistonen and Ms. Charlotte Roels for help with the practical work in the laboratory.

\section{References}

[1] D.E. Wurster, P.W. Taylor, Dissolution rates, Journal of pharmaceutical sciences 54(2) (1965) 169-175.

[2] A. Dokoumetzidis, P. Macheras, A century of dissolution research: from Noyes and Whitney to the biopharmaceutics classification system, International journal of pharmaceutics 321(1) (2006) 111.

[3] E.M. Merisko-Liversidge, G.G. Liversidge, Drug nanoparticles: formulating poorly watersoluble compounds, Toxicologic pathology 36(1) (2008) 43-48.

[4] G.L. Amidon, H. Lennernäs, V.P. Shah, J.R. Crison, A theoretical basis for a biopharmaceutic drug classification: the correlation of in vitro drug product dissolution and in vivo bioavailability, Pharmaceutical research 12(3) (1995) 413-420.

[5] A.A. Noyes, W.R. Whitney, The rate of solution of solid substances in their own solutions, Journal of the American Chemical Society 19(12) (1897) 930-934.

[6] D. Jünemann, J. Dressman, Analytical methods for dissolution testing of nanosized drugs, Journal of Pharmacy and Pharmacology 64(7) (2012) 931-943.

[7] J.L. Cohen, B.B. Hubert, L.J. Leeson, C.T. Rhodes, J.R. Robinson, T.J. Roseman, E. Shefter, The development of USP dissolution and drug release standards, Pharmaceutical research 7(10) (1990) 983-987. 
259 [8] J.B. Dressman, G.L. Amidon, C. Reppas, V.P. Shah, Dissolution testing as a prognostic tool for 260 oral drug absorption: immediate release dosage forms, Pharmaceutical research 15(1) (1998) 11-22.

261 [9] K.P. Shah, M. Chang, C.M. Riley, Automated analytical systems for drug development studies

262 3. Multivessel dissolution testing system based on microdialysis sampling, Journal of

263 pharmaceutical and biomedical analysis 13(10) (1995) 1235-1241.

264 [10] V. Pillay, R. Fassihi, Unconventional dissolution methodologies, Journal of pharmaceutical 265 sciences 88(9) (1999) 843-851.

266 [11] S.N. Bhattachar, J.A. Wesley, A. Fioritto, P.J. Martin, S.R. Babu, Dissolution testing of a 267 poorly soluble compound using the flow-through cell dissolution apparatus, International journal of 268 pharmaceutics 236(1) (2002) 135-143.

269

270

271

272

273

[12] S. Azarmi, W. Roa, R. Löbenberg, Current perspectives in dissolution testing of conventional and novel dosage forms, International Journal of pharmaceutics 328(1) (2007) 12-21.

[13] J. Shen, D.J. Burgess, In vitro dissolution testing strategies for nanoparticulate drug delivery systems: recent developments and challenges, Drug delivery and translational research 3(5) (2013) 409-415.

[14] C. Michalowski, S. Guterres, T. Dalla Costa, Microdialysis for evaluating the entrapment and release of a lipophilic drug from nanoparticles, Journal of pharmaceutical and biomedical analysis 35(5) (2004) 1093-1100.

[15] R. Peschka, C. Dennehy, F.C. Szoka Jr, A simple in vitro model to study the release kinetics of liposome encapsulated material, Journal of controlled release 56(1) (1998) 41-51.

[16] A. Sarnes, J. Østergaard, S.S. Jensen, J. Aaltonen, J. Rantanen, J. Hirvonen, L. Peltonen, Dissolution study of nanocrystal powders of a poorly soluble drug by UV imaging and channel flow methods, European Journal of Pharmaceutical Sciences 50(3) (2013) 511-519.

[17] D. Heng, D.J. Cutler, H.-K. Chan, J. Yun, J.A. Raper, What is a suitable dissolution method for drug nanoparticles?, Pharmaceutical research 25(7) (2008) 1696-1701.

[18] S.A. Qureshi, J. Shabnam, Cause of high variability in drug dissolution testing and its impact on setting tolerances, European Journal of Pharmaceutical Sciences 12(3) (2001) 271-276.

[19] S.A. Abouelmagd, B. Sun, A.C. Chang, Y.J. Ku, Y. Yeo, Release kinetics study of poorly water-soluble drugs from nanoparticles: are we doing it right?, Molecular pharmaceutics 12(3) (2015) 997-1003.

[20] C. Washington, Drug release from microdisperse systems: a critical review, International Journal of Pharmaceutics 58(1) (1990) 1-12.

[21] J.L. Baxter, J. Kukura, F.J. Muzzio, Hydrodynamics-induced variability in the USP apparatus II dissolution test, International journal of pharmaceutics 292(1) (2005) 17-28.

[22] D. D'Arcy, O. Corrigan, A. Healy, Hydrodynamic simulation (computational fluid dynamics) of asymmetrically positioned tablets in the paddle dissolution apparatus: impact on dis solution rate and variability, Journal of pharmacy and pharmacology 57(10) (2005) 1243-1250.

[23] G. Zhang, W. Vadino, T. Yang, W. Cho, I. Chaudry, Evaluation of the flow-through cell dissolution apparatus: effects of flow rate, glass beads and tablet position on drug release from different type of tablets, Drug development and industrial pharmacy 20(13) (1994) 2063-2078.

[24] P.P. Sanghvi, J.S. Nambiar, A.J. Shukla, C.C. Collins, Comparison of three dissolution devices for evaluating drug release, Drug development and industrial pharmacy 20(6) (1994) 961-980. 
301 [25] S. Modi, B.D. Anderson, Determination of drug release kinetics from nanoparticles:

302 overcoming pitfalls of the dynamic dialysis method, Molecular pharmaceutics 10(8) (2013) 30763033089.

304 [26] G. Moreno-Bautista, K.C. Tam, Evaluation of dialysis membrane process for quantifying the in 305 vitro drug-release from colloidal drug carriers, Colloids and Surfaces A: Physicochemical and 306 Engineering Aspects 389(1) (2011) 299-303.

307 [27] Ph.Eur., 4.01.03 Buffer solutions. , Eur. Pharmacopoeia Online 8.8. Available at:

308 http://online6.edqm.eu/ep808/ [Accessed August 16, 2016] (2015).

309 [28] E. Brunner, Reaktionsgeschwindigkeit in heterogenen Systemen, Z Phys Chem 43 (1904) 56310102.

311 [29] W. Nernst, Theorie der Reaktionsgeschwindigkeit in heterogenen Systemen, Zeitschrift für 312 Phys. Chemie, Stoechiom. und Verwandtschaftslehre 47 (1904) 52-55.

313 [30] W. Thomson, LX. On the equilibrium of vapour at a curved surface of liquid, The London, 314 Edinburgh, and Dublin Philosophical Magazine and Journal of Science 42(282) (1871) 448-452. 